\title{
Phenomenological studies of inclusive ep scattering at low momentum transfer $Q^{2}$
}

\author{
Bernd Surrow*†‡ \\ Deutsches Elektronen-Synchrotron DESY \\ Notkestr. 85, D-22607 Hamburg \\ E-mail: 'bernd. surrow@desy.dé
}

Abstract: Phenomenological studies on the proton structure function $F_{2}$ have been carried out to investigate the behavior of $F_{2}$, i.e. its $x$ and $Q^{2}$ dependence, in the transition from the deep-inelastic scattering region $\left(Q^{2} \gg 1 \mathrm{GeV}^{2}\right)$ to the photoproduction region $\left(Q^{2} \approx 0 \mathrm{GeV}^{2}\right)$. An overview of various results of $F_{2}$ at low $Q^{2}$ is given. To quantify the behavior of $F_{2}$ at low $Q^{2}$, the drivatives $d \ln \left(F_{2}\right) / d \ln (x)$ for fixed $Q^{2}$ and $d F_{2} / d \log \left(Q^{2}\right)$ for fixed $x$ have been determined and compared to expectations within the framework of perturbative and non-perturbative QCD. Furthermore, the derivatives were compared to results of the total inclusive diffractive cross-section and vector-meson production. An interpretation of the inclusive ep results is given in the framework of the GVD/CDP picture with special emphasis on the limit of $Q^{2} \rightarrow 0$ for fixed $x$ and $x \rightarrow 0$ for fixed $Q^{2}$.

\section{Introduction}

The measurement of the proton structure function $F_{2}$ at low $Q^{2}$ at HERA has attracted lots of interest and attention in recent years. The $\mathrm{H1}$ and ZEUS Collaborations have measured $F_{2}$ at low $Q^{2}$ with high precision using various experimental techniques. This allowed to study the behavior of $F_{2}$ in the transition from the deep-inelastic scattering region $\left(Q^{2} \gg 1 \mathrm{GeV}^{2}\right)$ to the photoproduction region $\left(Q^{2} \approx 0 \mathrm{GeV}^{2}\right)$.

At low $Q^{2}$ a perturbative QCD (pQCD) approach based on NLO DGLAP fits is expected to lose its validity for fundamental reason. This is due to the underlying nonAbelian nature of the QCD field theory and its corresponding behavior of the coupling constant $\alpha_{s}$ to be large at large distances which therefore does not permit a perturbative approach. Thus other non-perturbative QCD concepts have to be considered such as Regge

\footnotetext{
${ }^{*}$ Speaker.

${ }^{\dagger}$ Now at Brookhaven National Laboratory.

${ }^{\ddagger}$ On behalf of the H1 and ZEUS Collaborations.
} 


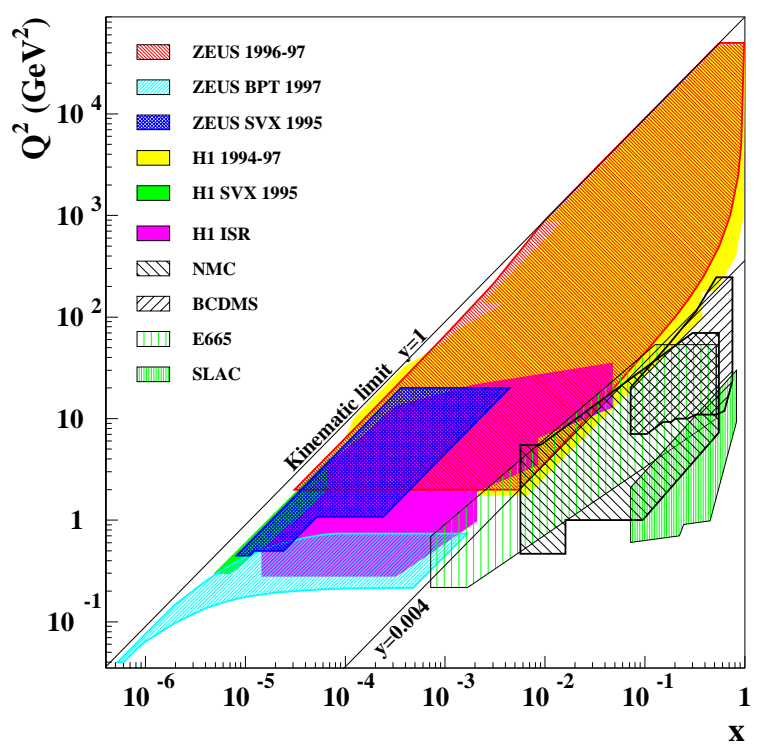

Figure 1: Kinematic coverage in the $Q^{2}-x$ plane for various fixed-target experiments and the HERA collider experiments H1 and ZEUS.

phenomenology, Generalized Vector Dominance (GVD) and Color Dipole Picture (CDP) model approaches to name only the most prominent.

It has been shown that a pQCD analysis based on NLO DGLAP fits allow to describe the behavior of $F_{2}$ down to approximately $Q^{2} \approx 1 \mathrm{GeV}^{2}[1]$. Although the quality of those fits are still acceptable down to $Q^{2} \approx 1 \mathrm{GeV}^{2}$, various expectations on the behavior of the underlying parton distributions at high $Q^{2}$ are no longer valid at low $Q^{2}$, e.g. the behavior of the gluon distribution. This gave rise to several theoretical debates on the validity of NLO DGLAP fits at low $Q^{2}$ indicating that the limits of a pQCD approach are reached earlier than simply given by the quality of the NLO DGLAP fits. Putting it in other words, the application of a partonic picture such as pQCD based on NLO DGLAP fits cannot necessarily be pushed down to $Q^{2} \approx 1 \mathrm{GeV}^{2}$ without loosing its physical interpretation. At low $Q^{2}$ and in particular in the limit of $Q^{2} \rightarrow 0$ - which is a well known fact - a hadronic behavior becomes important which can be well accounted for by Regge phenomenology.

It has recently been shown that the GVD/CDP model approach provides at low $x$ a successful description of $F_{2}$ from the deep-inelastic scattering region $\left(Q^{2} \gg 1 \mathrm{GeV}^{2}\right)$ to the photoproduction region $\left(Q^{2} \approx 0 \mathrm{GeV}^{2}\right)$. It still remains to be shown how these findings can be interpreted based on a microscopic picture of quark and gluon dynamics. Such an attempt has recently been published in [i]

The next section will provide an overview of various $F_{2}$ results showing the $Q^{2}$ and $x$ dependence of $F_{2}$ as well as the $Q^{2}$ and $W^{2}$ dependence of the total $\gamma^{*} p$ cross-section $\sigma_{\text {tot }}^{\gamma^{*} p}$ which is in particular important when approaching the photoproduction limit $\left(Q^{2} \rightarrow 0\right)$.

To quantify the behavior of $F_{2}$ at low $Q^{2}$, the derivatives $d \ln \left(F_{2}\right) / d \ln (x)$ for fixed $Q^{2}$ and $d F_{2} / d \log \left(Q^{2}\right)$ for fixed $x$ have been determined and compared to expectations within the framework of perturbative and non-perturbative QCD. Furthermore, the derivatives were compared to results of the total inclusive diffractive cross-section and vector-meson 


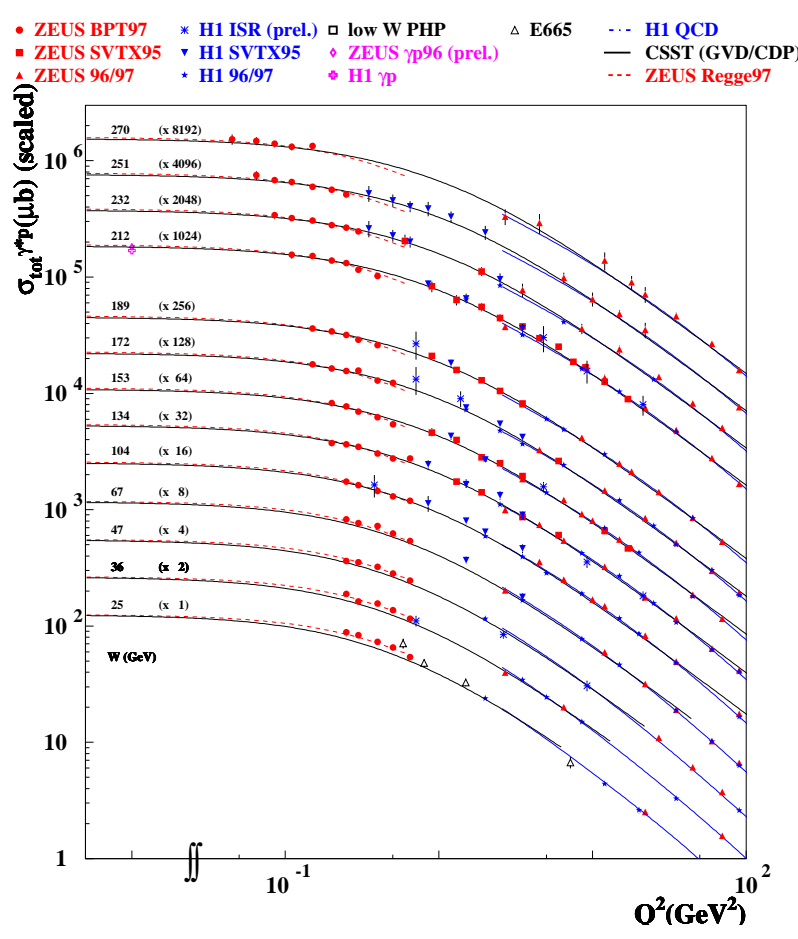

Figure 2: $\sigma_{\text {tot }}^{\gamma^{*} p}$ as a function of $Q^{2}$ for different values of $W$.

production. Those results will be shown in section 3 .

An attempt is made in section 4 to interpret the HERA inclusive ep scattering results in the framework of the GVD/CDP model approach. It will be shown that a universal hadronic behavior of the total $\gamma^{*} p$ cross-section is reached not only in the limit of $Q^{2} \rightarrow 0$ for fixed $W^{2}$ which is seen at HERA but also - without experimental verification yet - in the limit of $x \rightarrow 0$ for fixed $Q^{2}$, i.e. $\sigma_{\text {tot }}^{\gamma^{*} p} \rightarrow \sigma_{\text {tot }}^{\gamma p}$.

\section{Overview plots of $F_{2}$ and $\sigma_{\text {tot }}^{\gamma^{*} p}$}

Figure ${ }_{1}^{1}$ shows the kinematic coverage in the $Q^{2}-x$ plane for various fixed-target exper-

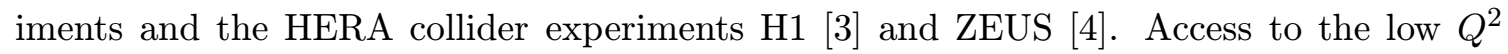
region was made possible using various experimental techniques. A dedicated detector to measure the scattered electron under very small angles and thus to provide acceptance at low $Q^{2}$ has been installed within the ZEUS experiment. This effort allowed to measure $F_{2}$ over a wide range in $x$ and $Q^{2}$ with high precision (ZEUS BPT 1997). Dedicated HERA runs with a shifted event vertex compared to the nominal interaction vertex have been used at H1 and ZEUS to effectively gain acceptance at low $Q^{2}$ (H1 SVX 1995 and ZEUS SVX 1995). Preliminary results on a measurement of $F_{2}$ at low $Q^{2}$ using initial-state radiation events thus using events with a lower center-of-mass energy and thereby gaining acceptance at low $Q^{2}$ has been reported by the H1 Collaboration (H1 ISR).

Those measurements allowed an experimental basis to examine the behavior of $F_{2}$ at low $Q^{2}$ over a wide range in $x$. Taking all these measurements together overview plots of 
the $Q^{2}$ and $x$ dependence of $F_{2}$ as well as the $Q^{2}$ and $W^{2}$ dependence of the total $\gamma^{*} p$ cross-section $\sigma_{\text {tot }}^{\gamma^{*} p}$ are shown in Figures

Figure $\overline{2}$ shows the total $\gamma^{*} p$ cross-section as a function of $Q^{2}$ for different values of $W$. Also shown is the measurement of the total photoproduction cross-section by the H1 and ZEUS Collaborations. A steady decrease of $\sigma_{\text {tot }}^{\gamma^{*} p}$ with increasing $Q^{2}$ for $Q^{2}>1 \mathrm{GeV}^{2}$ can be seen which is well accounted for by NLO DGLAP-fits [3i] (H1 97). Going towards lower values of $Q^{2}$ a characteristic change of $\sigma_{\text {tot }}^{\gamma^{*} p}$ is visible at around $Q^{2} \approx 1 \mathrm{GeV}^{2}$. The

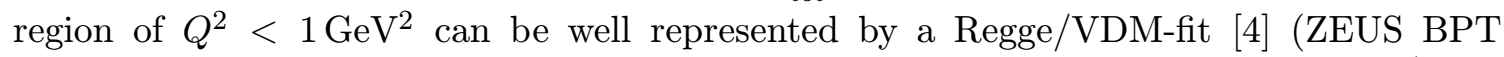

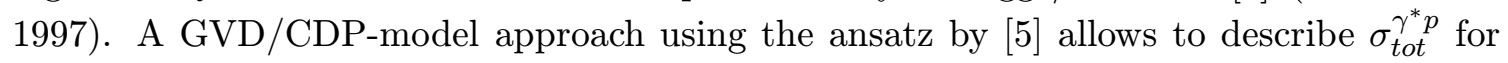
$Q^{2}<100 \mathrm{GeV}^{2}$ and $x<0.01$.

Figure $13 \overline{3}$ shows the total $\gamma^{*} p$ cross-section as a function of $W^{2}$ for different values of $Q^{2}$. $\sigma_{\text {tot }}^{\gamma^{*} p}$ is seen to increase more strongly with $W^{2}$ for larger values of $Q^{2}$ which is well described by NLO DGLAP-fits [i] (H1 97). At low $Q^{2}$ and thus towards the photoproduction limit the milder increase of $\sigma_{\text {tot }}^{\gamma^{*} p}$ can be represented by a Regge/VDM-fit [i $\left[\mathbf{A}_{i}\right]$ (ZEUS BPT 1997). For $x<0.01$ and $Q^{2}<100 \mathrm{GeV}^{2}$ the GVD/CDP-model approach [īin] provides a good description of the $W^{2}$ dependence.

Figure ${ }_{1}^{4}$ shows $F_{2}$ as a function of $Q^{2}$ for different values of $x . F_{2}$ diminishes towards low $Q^{2}$ like $F_{2} \propto Q^{2} \rightarrow 0$ for $Q^{2} \rightarrow 0$. The rise of $F_{2}$ with $Q^{2}$ increases in the low $x$ region which is larger at high $Q^{2}$ and smaller at low $Q^{2}$. The region of $Q^{2}<1 \mathrm{GeV}^{2}$ can be well described by a Regge/VDM-fit [4] (ZEUS BPT 1997) whereas the region for $Q^{2}>1 \mathrm{GeV}^{2}$ can be well accounted for by NLO-DGLAP fits [i3i] (H1 97). For $x<0.01$ and

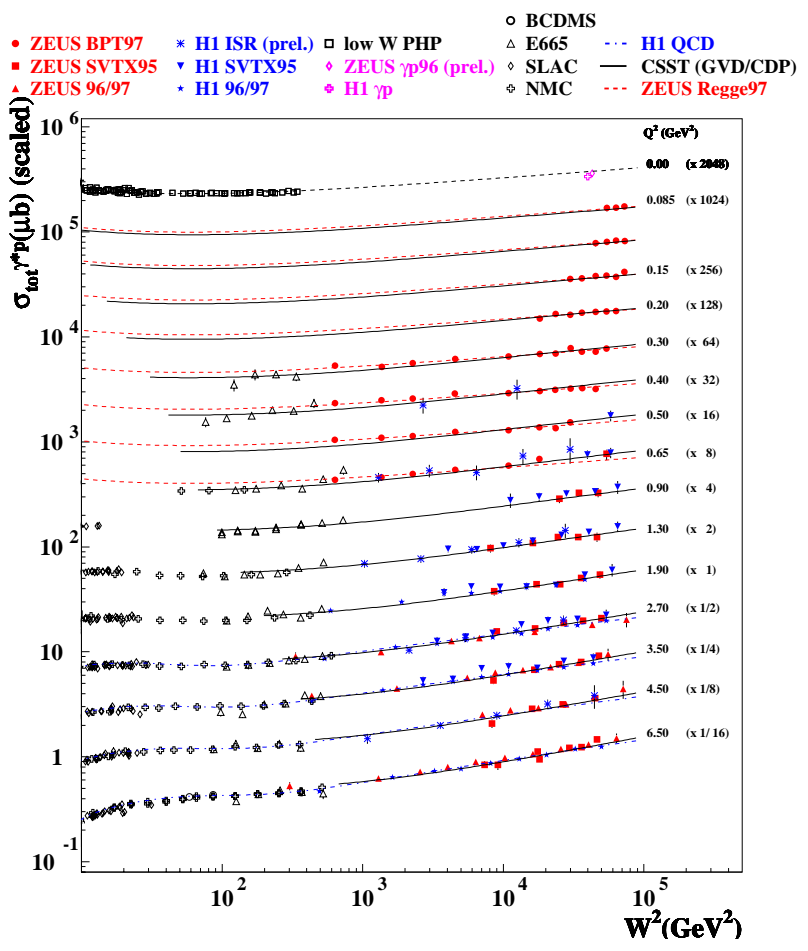

Figure 3: $\sigma_{\text {tot }}^{\gamma^{*} p}$ as a function of $W^{2}$ for different values of $Q^{2}$. 


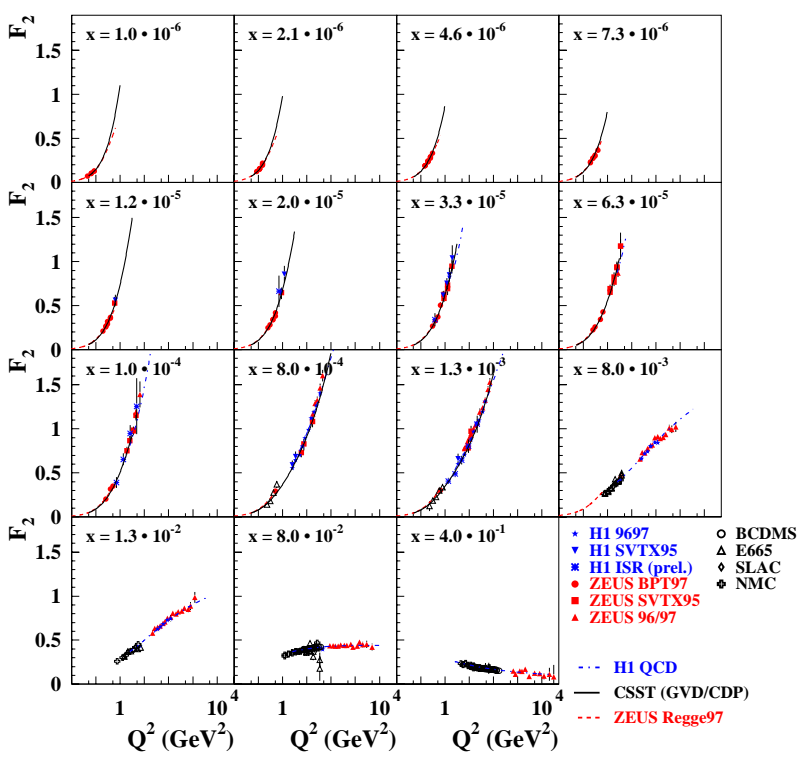

Figure 4: $F_{2}$ as a function of $Q^{2}$ for different values of $x$.

$Q^{2}<100 \mathrm{GeV}^{2}$ the GVD/CDP-model approach [5] provides a good description of the $Q^{2}$ dependence.

Figure ${ }_{-1}^{-1}$ shows $F_{2}$ as a function of $x$ for different values of $Q^{2}$. The rise of $F_{2}$ diminishes towards low $Q^{2}$ and reaches the expected $x$-dependence which is well accounted for by a Regge/VDM-description [4i-i $]$ (ZEUS BPT 1997) based on a soft Pomeron ansatz. Towards larger values of $Q^{2}$ NLO DGLAP-fits [3i] (H1 97) successfully describe the $x$-dependence of $F_{2}$. For $x<0.01$ and $Q^{2}<100 \mathrm{GeV}^{2}$ the GVD/CDP-model approach provides a good description of the $x$ dependence.

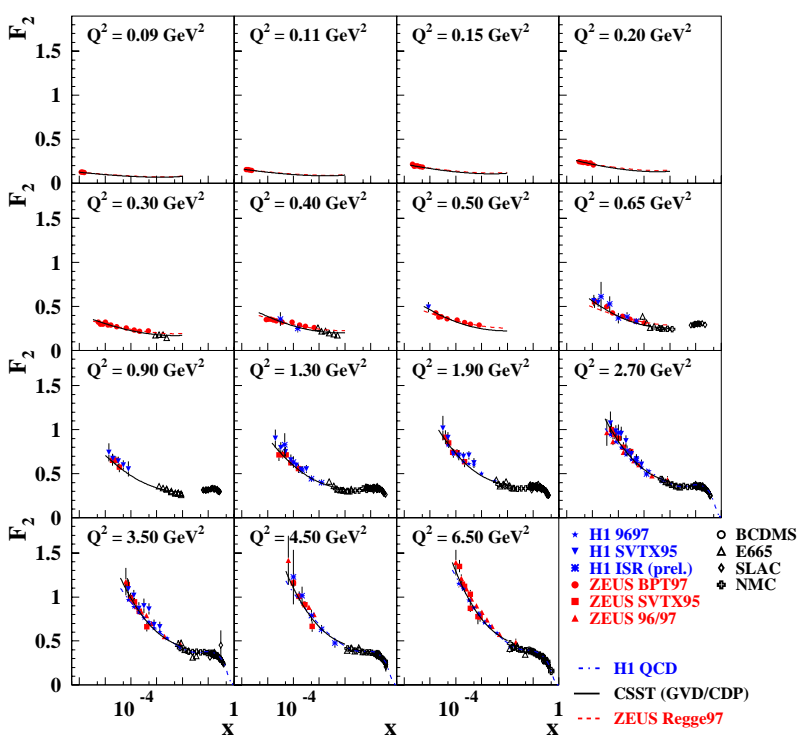

Figure 5: $F_{2}$ as a function of $x$ for different values of $Q^{2}$. 


\section{Phenomenological studies on the $x$ and $Q^{2}$ dependence}

Both, the H1 and ZEUS Collaborations have carried out phenomenological studies to quantify the observed behavior of $F_{2}$ at low $Q^{2}$ which was discussed in the last section. The derivatives $d \ln \left(F_{2}\right) / d \ln (x)$ for fixed $Q^{2}$ and $d F_{2} / d \log \left(Q^{2}\right)$ for fixed $x$ have been determined and compared to expectations within the framework of perturbative and non-perturbative QCD.

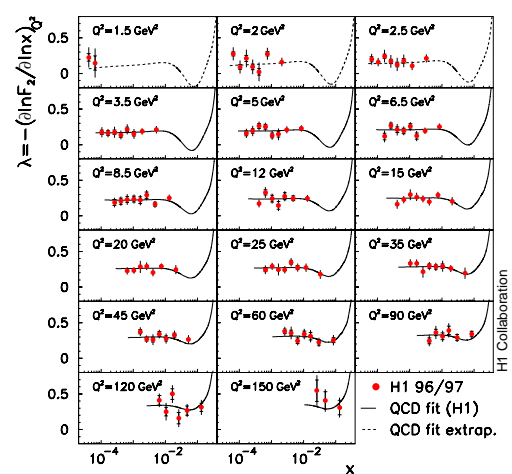

Figure 6: $H 1$ results on the measurement of the function $\lambda\left(x, Q^{2}\right)$ as a function of $x$ for different values of $Q^{2}$.

The H1 Collaboration extracted for the fist time the derivative $\left(\frac{\partial \ln F_{2}\left(x, Q^{2}\right)}{\partial \ln x}\right)_{Q^{2}} \equiv$

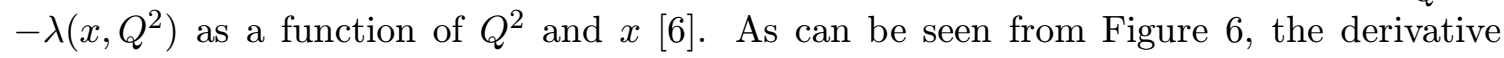
$\lambda\left(x, Q^{2}\right)$ is found to be independent of $x$ for $x<0.01$. This implies that the $x$ dependence of $F_{2}$ at low $x$ is consistent with a power-law behavior, $F_{2} \propto x^{-\lambda}$, for fixed values of $Q^{2}$. Furthermore the rise of $F_{2}$ at low $x$ which was one of the first observations at HERA, is found to be proportional to $F_{2} / x$. Thus, there is no evidence for a change in this behavior in the measured kinematical region. The observed behavior at low $x$ is consistent with the result of a pQCD fit.

These findings justify that the behavior of $F_{2}$ can be simply quantified as $F_{2}=C\left(Q^{2}\right)$. $x^{-\lambda\left(Q^{2}\right)}$. Figure $\bar{T}_{1}$ shows the exponent $\lambda\left(Q^{2}\right)$ as a function of $Q^{2} . \lambda\left(Q^{2}\right)$ is seen to rise linearly with $\ln Q^{2}$. The extracted coefficient $C\left(Q^{2}\right)$ is found to be independent of $Q^{2}$ within experimental uncertainties.

A similar analysis extracting $\lambda\left(Q^{2}\right)$ as a function of $x$ has been carried out by the ZEUS Collaboration. The result of this preliminary analysis is shown in Figure ${ }_{-i}^{\overline{8}}$. The extracted values for $\lambda\left(Q^{2}\right)$ extend to lower values in $Q^{2}$. The results agree with the ZEUS QCD01 parameterization down to $Q^{2}=2.5 \mathrm{GeV}^{2}$. For $Q^{2}<0.6 \mathrm{GeV}^{2}, \lambda\left(Q^{2}\right)$ is consistent with a constant value of $\approx 0.1$ as expected from the assumption of a single soft Pomeron exchange within the framework of Regge phenomenology. Thus the slope in $F_{2}$ at low $x$ and very low $Q^{2}$ is found to be independent of $Q^{2}$ within experimental uncertainties. This behavior can be also inferred from Figure $\underline{3}$. The $\gamma^{*} p$ cross-section at fixed values of $W^{2}$ saturates for $Q^{2} \rightarrow 0$.

An attempt was made to compare the energy dependence of the inclusive $\gamma^{*} p$ crosssection which is equivalent to the above studies on the $x$ dependence of $F_{2}$, to the energy 


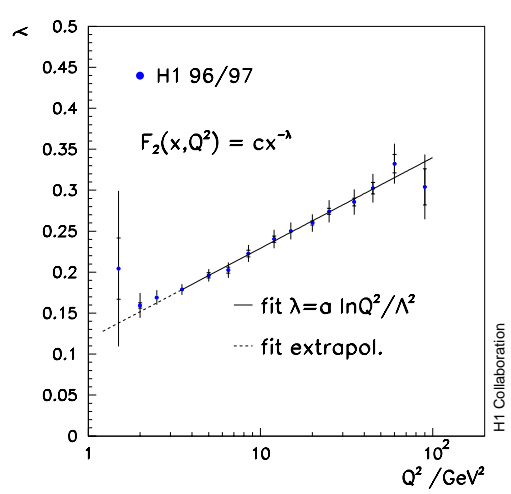

Figure 7: $H 1$ results on $\lambda\left(Q^{2}\right)$ as a function of $Q^{2}$ extracted from a fit of the form $F_{2}=C\left(Q^{2}\right)$. $x^{-\lambda\left(Q^{2}\right)}$.

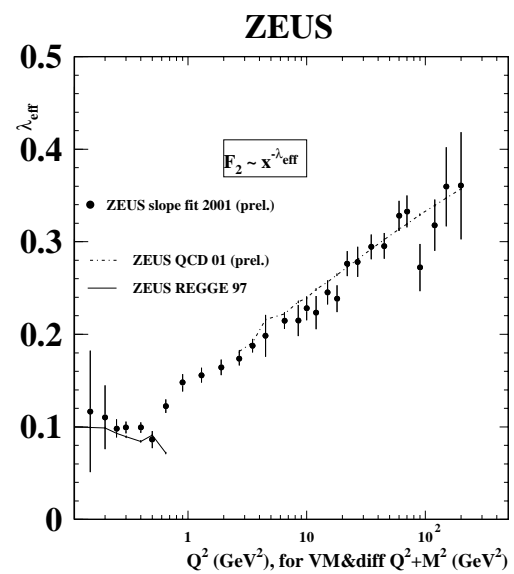

Figure 8: ZEUS preliminary results on $\lambda_{\text {eff }}=\lambda\left(Q^{2}\right)$ as a function of $Q^{2}$. The estimates of the ZEUS Regge97 and ZEUS QCD01 parameterizations are also shown.

dependence of the total inclusive diffractive cross-section (Diff), vector meson production (VM) and deeply virtual Compton scattering (DVCS) at HERA. The total $\gamma^{*} p$ crosssection can be related to the elastic vector meson cross-section using the Optical Theorem which implies that $\sigma_{\text {tot }}^{\gamma^{*} p}$ varies with $W^{2}$ as $\sigma_{\text {tot }}^{\gamma^{*} p} \propto\left(W^{2}\right)^{\epsilon}$ whereas the elastic vector meson cross-section varies with $W^{2}$ as $\sigma_{e l}\left(\gamma^{*} p \rightarrow V p\right) \propto\left(W^{2}\right)^{2 \epsilon}$.

Figure $\bar{q}_{1}$ shows a compilation of various slope analyses from the H1 and ZEUS Collaborations. The inclusive results which were discussed earlier are found to be consistent within experimental uncertainties. The vector meson analysis indicated that the equivalent slope, $\lambda_{V M}$, scales with $Q^{2}+M_{V M}^{2}$ where $M_{V M}$ is the vector meson mass. The extracted values for $\lambda_{D i f f}, \lambda_{V M}$ and $\lambda_{D V C S}$ are multiplied by a factor $1 / 2$ in the comparison to the inclusive results.

The naive expectations of $\lambda_{V M} / 2=\lambda$ is approximately true. $\lambda_{D i f f} / 2$ is somewhat below the inclusive result indicating that the inclusive and inclusive diffractive cross-sections have the same $W$ dependence contrary to the case of elastic vector-meson production.

The large uncertainties however do not permit to draw any further conclusions yet.

Both, the H1 and ZEUS Collaborations, extracted besides the $x$ slope of $F_{2}$ also the 


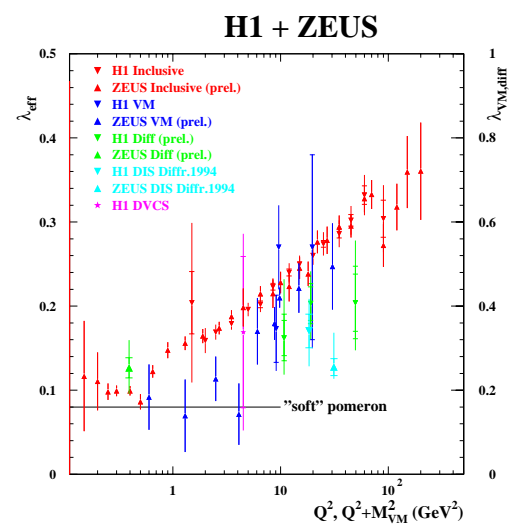

Figure 9: Compilation of various slope analyses from the H1 and ZEUS Collaborations on the energy dependence of the respective cross-sections: inclusive results (Inclusive), vector-meson results (VM), inclusive diffractive results (Diff) and deeply-virtual Compton scattering results (DVCS).

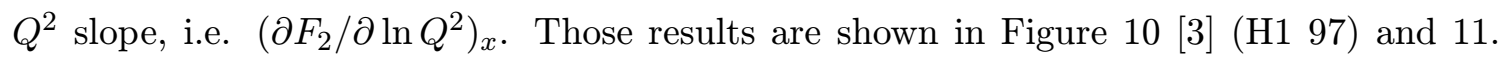
A consistent picture of the H1 and ZEUS analysis is obtained on the $Q^{2}$ slope of $F_{2}$ as a function of $x$. At low $Q^{2}$, the slope of $F_{2}$ increases only slowly towards low values of $x$ whereas at high $Q^{2}$ it increases rather strongly. This behavior can be also inferred from

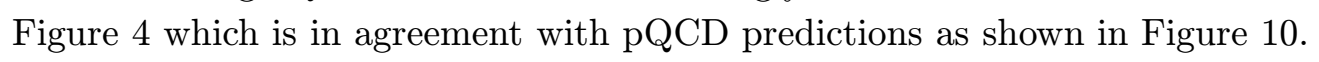

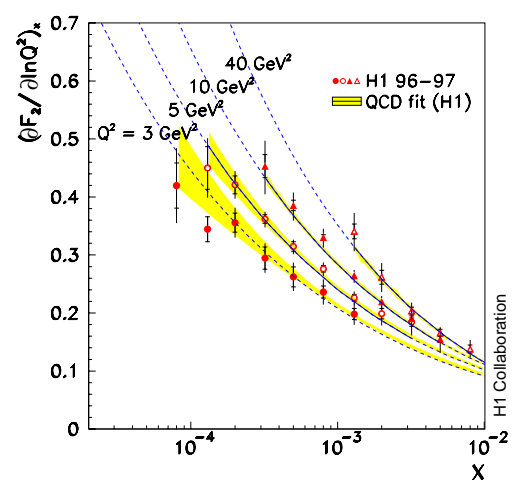

Figure 10: $H 1$ results on $\left(\partial F_{2} / \partial \ln Q^{2}\right)_{x}$ as function of $x$ for different values of $Q^{2}$. The prediction from a $Q C D$-fit is also shown.

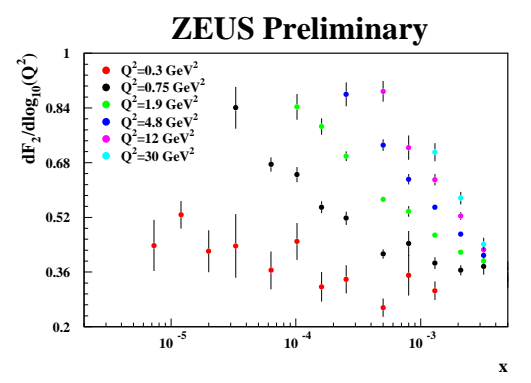

Figure 11: ZEUS preliminary results on $\left(\partial F_{2} / \partial \ln Q^{2}\right)_{x}$ as function of $x$ for different values of $Q^{2}$. 


\section{Interpretation of HERA data}

The $W^{2}$ and $Q^{2}$ dependence of the total $\gamma^{*} p$ cross-section has been discussed in detail in the last section.

To summarize, the steep rise of the inclusive $\gamma^{*} p$ cross-section with increasing $W^{2}$ which is equivalent to the steep rise of $F_{2}$ towards low $x$, diminishes for $Q^{2} \rightarrow 0$ and reaches the energy dependence of the total $\gamma p$ cross-section. At low $Q^{2}$, the cross-section behavior of the total inclusive $\gamma^{*} p$ cross-section can be well accounted for by Regge phenomenology whereas at high $Q^{2}$, pQCD based on a NLO DGLAP analysis allows to describe the observed behavior.

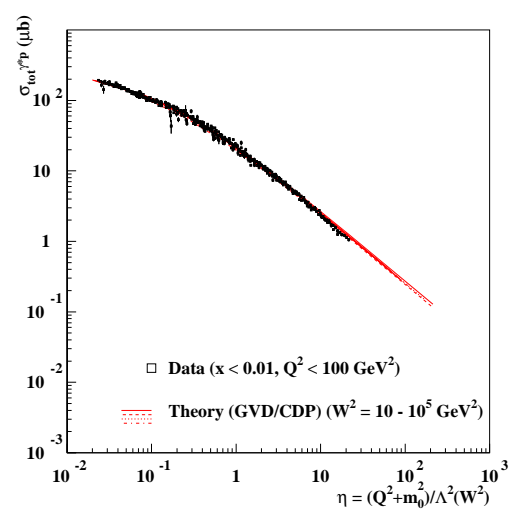

Figure 12: The experimental data for $\sigma_{\text {tot }}^{\gamma^{(*)}}\left(W^{2}, Q^{2}\right)$ for $x<0.01$ including photoproduction results as a function of the scaling variable $\eta=\left(Q^{2}+m_{0}^{2}\right) / \Lambda\left(W^{2}\right)$.

At low $x$ and $Q^{2}<100 \mathrm{GeV}^{2}$ it was shown that a GVD/CDP-model approach allows to describe the observed cross-section behavior. In the formulation of the above model by [5] besides others [i] show a scaling behavior of the form $\sigma_{\text {tot }}^{\gamma^{(*)}} p\left(W^{2}, Q^{2}\right)=\sigma_{t o t}^{\gamma^{(*)}} p(\eta)$ with $\eta=\left(Q^{2}+m_{0}^{2}\right) / \Lambda\left(W^{2}\right)$. The scale $\Lambda^{2}\left(W^{2}\right)$ turned out to be an increasing function of $W^{2}$ and my be represented by a power law $\left(\Lambda\left(W^{2}\right)=C_{1}\left(W^{2}+W_{0}^{2}\right)_{2}^{C}\right)$ or logarithmic $\left(\Lambda\left(W^{2}\right)=C_{1}^{\prime} \ln \left(W^{2} / W_{0}^{2}+C_{2}^{\prime}\right)\right)$ function of $W^{2}$. The threshold mass $m_{0}^{2}$ as well as the other three parameters of the function $\Lambda^{2}\left(W^{2}\right)$ have been constrained by the data itself. The result of this analysis can be seen in Figure ${ }_{1}^{1} 2_{-}^{1}$ It has been shown that this scaling behavior improves with decreasing $x$. The HERA data on DIS in the low $x$ diffraction region, including photoproduction, find a natural interpretation in the GVD/CDP picture that rests on the generic structure of two-gluon exchange from QCD.

If one assumes that this behavior continues at extremely low $x$ or large values of $W^{2}$ one is faced to conclude that $\sigma_{\text {tot }}^{\gamma^{*} p}$ reaches the energy behavior of the total photoproduction cross-section. Thus there are two limits which allows the total $\gamma^{*} p$ cross-section to saturate. First, for fixed values of $W^{2}$, one finds $\sigma_{\text {tot }}^{\gamma^{*} p} \rightarrow \sigma_{\text {tot }}^{\gamma p}$ which has been clearly seen at HERA (Figure ${ }_{2}^{2}$ ). The second limit of $x \rightarrow 0$ or equivalently of $W^{2} \rightarrow \infty$ at fixed $Q^{2}$ can be inferred from the above findings within the GVD/CDP picture. The total $\gamma^{*} p$ cross-section reaches for $W^{2} \rightarrow \infty$ the total photoproduction cross-section limit for any fixed values of 
$Q^{2}$. The second limit however has not been experimentally confirmed so far and it will be rather difficult in the future to do so. Figure $1 \overline{3}$ in shows expectations for the behavior of the total $\gamma^{*} p$ cross-section at large $W^{2}$ using the derived analytical expression for $\sigma_{\text {tot }}^{\gamma^{*} p}$ by [.5.5. This demonstrates clearly the asymptotic behavior $\sigma_{\text {tot }}^{\gamma^{*} p} / \sigma_{\text {tot }}^{\gamma p} \rightarrow 1$ for $W^{2} \rightarrow \infty$. The kinematical region of HERA is also shown which is far from the second limit described above.

In summary, one is led to conclude based on the findings within the GVD/CDP picture that the real and virtual photons on protons become identical in the limit of infinite energy.

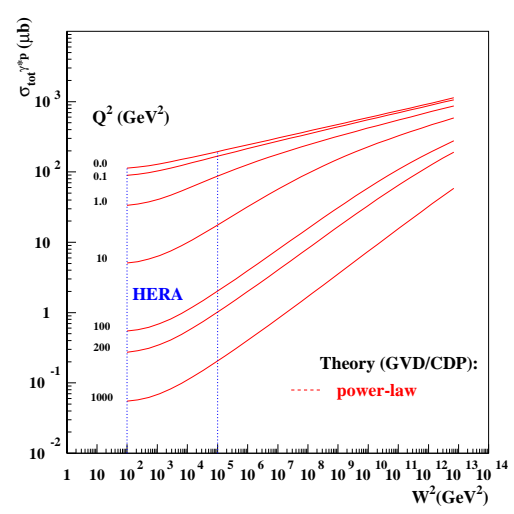

Figure 13: Expectations of the cross-section behavior of the total $\gamma^{*} p$ cross-section at large $W^{2}$ using the derived analytical expression for $\sigma_{\text {tot }}^{\gamma^{(*)} p}$ by [is]

\section{References}

[1] H1 97: H1 Collab., C. Adloff et al., Eur. Phys. J. C13 (2000) 609; ZEUS Collab., J. Breitweg et al., Eur. Phys. J. C7 (1999) 609;

B. Surrow, EPJdirect C2 (1999) 1.

[2] D. Schildknecht, B. Surrow and M. Tentyukov, Mod. Phys. Lett. A16 (2001) 1829.

[3] H1 SVTX 95: H1 Collab., C. Adloff et al., Nucl. Phys. B497 (1997) 3; H1 97: H1 Collab., C. Adloff et al., Eur. Phys. J. C13 (2000) 609.

[4] ZEUS SVTX 95: ZEUS Collab., J. Breitweg et al., Eur. Phys. J. C7 (1999) 609; ZEUS BPT 97: ZEUS Collab., J. Breitweg et al., Phys. Lett. B487 (2000) 53; ZEUS 9697: ZEUS Collab., S. Chekanov et al., Eur. Phys. J. C21 (2001) 443.

[5] G. Cvetic, D. Schildknecht, B. Surrow and M. Tentyukov, Eur. Phys. J. C20 (2001) 77;

D. Schildknecht, B. Surrow and M. Tentyukov, Phys. Lett. B499 (2001) 116.

[6] H1 Collab., C. Adloff et al., Phys. Lett. B520 (2001) 183.

[7] J. Forshaw, G. Kerley and G. Shaw, Phys. Rev. D60 (1999) 074012;

K. Golec-Biernat and M. Wüsthoff, Phys. Rev. D59 (1999) 014017;

K. Golec-Biernat and M. Wüsthoff, Phys. Rev. D60 (1999) 114023. 